\title{
Timely Cancer Genetic Counseling and Testing for Young Women With Breast Cancer: Impact on Surgical Decision-making for Contralateral Risk- reducing Mastectomy
}

\section{Shenin Dettwyler ( $\nabla$ s.a.dettwyler@gmail.com )}

NYU Langone Health https://orcid.org/0000-0001-9391-3471

Darcy Thull

Magee-Womens Hospital of UPMC: UPMC Magee-Womens Hospital

Priscilla McAuliffe

Magee-Womens Hospital of UPMC: UPMC Magee-Womens Hospital Jennifer Steiman

Magee-Womens Hospital of UPMC: UPMC Magee-Womens Hospital

Ronald Johnson

Magee-Womens Hospital of UPMC: UPMC Magee-Womens Hospital

Emilia Diego

Magee-Womens Hospital of UPMC: UPMC Magee-Womens Hospital

Phuong Mai

University of Pittsburgh School of Medicine

\section{Research Article}

Keywords: breast cancer, genetic counseling, genetic testing, risk-reducing mastectomy, variant of uncertain significance

Posted Date: January 5th, 2022

DOI: https://doi.org/10.21203/rs.3.rs-1146819/v1

License: (c) (1) This work is licensed under a Creative Commons Attribution 4.0 International License. Read Full License 
Timely Cancer Genetic Counseling and Testing for Young Women with Breast Cancer: Impact on Surgical Decision-Making for Contralateral Risk-Reducing Mastectomy

Shenin A. Dettwyler, $\mathrm{MS}^{1 *}$; Darcy L. Thull, $\mathrm{MS}^{1}$; Priscilla F. McAuliffe, MD, PhD²; Jennifer G. Steiman, $\mathrm{MD}^{2}$; Ronald R. Johnson, MD²; Emilia J. Diego, MD²; Phuong L. Mai, MD, MS ${ }^{3}$

${ }^{1}$ UPMC Magee-Womens Hospital (Cancer Genetics Program), Pittsburgh, PA, USA

${ }^{2}$ UPMC Magee-Womens Hospital (Magee-Womens Surgical Associates), Pittsburgh, PA, USA

${ }^{3}$ University of Pittsburgh School of Medicine (Center for Clinical Genetics and Genomics), Pittsburgh, PA, USA

*Currently at NYU Langone Health (The Pancreatic Cancer Center), New York, NY, USA

Corresponding author: Shenin A. Dettwyler, MS; shenin.dettwyler@nyulangone.org

ORCID IDs: Shenin Dettwyler, 0000-0001-9391-3471

Phuong Mai, 0000-0002-7486-4974

Darcy Thull, 0000-0001-7999-2804

Priscilla McAuliffe, 0000-0002-2933-1191

Emilia Diego, 0000-0003-2155-817X 


\begin{abstract}
PURPOSE: Genetic testing (GT) can identify individuals with pathogenic variants (PV) in breast cancer (BC) predisposition genes, who may consider contralateral risk-reducing mastectomy (CRRM). We report on CRRM rates in young women newly diagnosed with BC who received GT through a multidisciplinary clinic.
\end{abstract}

METHODS: Clinical data was reviewed for patients seen between November 2014 and June 2019. Patients with non-metastatic, unilateral BC diagnosed at age $\leq 45$ and completed GT prior to surgery were included. Associations between surgical intervention and age, BC stage, family history, and GT results were evaluated.

RESULTS: Of the 194 patients, $30(15.5 \%)$ had a PV in a BC predisposition gene (ATM, BRCA1, BRCA2, CHEK2, $N B N, N F 1)$, with $66.7 \%$ in $B R C A 1$ or BRCA2. Of $164(84.5 \%)$ uninformative results, $132(68 \%)$ were negative and $32(16.5 \%)$ were variants of uncertain significance (VUS). Overall, 67 (34.5\%) had CRRM, including 25/30 (83.3\%) PV carriers and 42/164 (25.6\%) non-carriers. Only a positive test result was associated with CRRM $(p<0.01)$. For the 164 with uninformative results, CRRM was not associated with age $(p=0.23)$, a VUS, $(p=0.08)$, family history $(p=0.19)$, or BC stage $(p=0.10)$.

CONCLUSION: In this cohort of young women with BC, the identification of a PV in a BC predisposition gene was the only factor associated with the decision to pursue CRRM. Thus, incorporation of genetic services in the initial evaluation of young patients with a new BC could contribute to the surgical decision-making process.

Keywords: breast cancer, genetic counseling, genetic testing, risk-reducing mastectomy, variant of uncertain significance 
TIMELY CANCER GENETIC COUNSELING AND TESTING YOUNG WOMEN WITH BREAST CANCER

\section{INTRODUCTION}

Breast cancer $(\mathrm{BC})$ is the most common cancer diagnosis for women in the United States.[1] The average woman has an approximately $12.9 \%$ lifetime risk of developing BC, with a median diagnosis age of 62 years.[1] Approximately 5-10\% of BC are attributable to pathogenic variants (PVs) in a BC predisposition gene.[2, 3] PVs in BRCA1 and BRCA2 (BRCA1/2) are the most common cause of hereditary BC, and are associated with a lifetime BC risk of up to 69-72\%.[4] Lifetime BC risks associated with other BC predisposition genes vary from at least a 4-fold increase for the high-risk genes (CDH1, PALB2, PTEN, STK11, and TP53) to a 2- to 3-fold increase for the moderate-risk genes (ATM, $C H E K 2, N B N$, and NF1).[4-12] Breast cancer associated with a hereditary predisposition may also be diagnosed at earlier ages; in particular, women with BRCA1/2 or TP53 PVs are often diagnosed with premenopausal BC.[4, 13]

Contralateral BC risks are influenced by multiple factors, including age at first BC diagnosis, family history of $\mathrm{BC}$, previous treatments, and underlying genetic predisposition. [4, 14-18] The risk for a contralateral BC is approximately $10 \%$ within 20 years after the initial diagnosis for unselected women with no known hereditary BC predispositions, [18] but may be as high as 53-65\% for BRCA1/2 PV carriers.[4, 19] Contralateral BC risks have not been well-defined for most other BC predisposition genes, but may be increased for women with ATM, PALB2, TP53, and CHEK2 truncating PVs.[13, 20-23]

Genetic testing (GT) is recommended for women with newly diagnosed BCs who meet specific criteria based on their personal and family cancer history, as the identification of a hereditary predisposition may impact care.[24-27] Women with PVs in high-risk genes with available data on contralateral BC risks could consider contralateral risk-reducing mastectomy $(\mathrm{CRRM})$ at the time of surgical $\mathrm{BC}$ treatment, which provides a $>90 \%$ reduction in future $\mathrm{BC}$ risk.[23, 27-29] However, CRRM is not routinely recommended for women without a BC predisposition, or for women with PVs in moderate-risk genes with insufficient data regarding second primary BC risks or long-term benefits of CRRM.[23, 28, 30-32]

Despite the limited data on survival benefits of CRRM, CRRM rates have been increasing in United States over the past two decades across cohorts of women with unilateral BC treated in various geographical locations between the mid-1990s to early 2010s. The increase in CRRM rates ranges from of $4.2 \%$ to $9.6 \%$ over 8 years, to an increase of $5.4 \%$ to $37.5 \%$ over 15 years.[23, 33-39] 
TIMELY CANCER GENETIC COUNSELING AND TESTING YOUNG WOMEN WITH BREAST CANCER

Genetic testing availability has also increased over the past few decades, including increased utilization of multigene panel testing, which has increased the PV detection rates in genes other than BRCA1/2.[40-42] However, limited information exists regarding the impact of PVs in other BC predisposition genes on surgical decision-making outcomes for BC patients.[23, 43-46]

Regarding GT results, it is also important to consider the impact of variants of uncertain significance (VUSs) on surgical decision-making. As VUSs do not carry any clinical implications, patients with a VUS should follow management recommendations based on their personal and family history, rather than on the GT result.[27, 47, 48] However, VUS identification can lead to patients being recommended the same management considerations as PV carriers, including CRRM.[49-53]

Given broader GT availability and subsequent improvements in the ability to identify PVs in BC predisposition genes other than $B R C A 1 / 2$ in recent years, we sought to further explore the impact of GT on surgical decision-making. This study focused on a cohort of young female patients with a new diagnosis of unilateral BC, who were uniformly offered genetic counseling and GT as part of a multidisciplinary clinic designed to efficiently provide patients with comprehensive care at the time of diagnosis.

\section{METHODS}

Clinical data for patients seen in the multidisciplinary Breast Cancer Specialty Care Clinic at MageeWomens Hospital of UPMC (Pittsburgh, PA) between November 2014 and June 2019 were abstracted. This chart review was performed under a Quality Improvement project to evaluate the utility of this clinic for young breast cancer patients. The project was reviewed and approved by the University of Pittsburgh Medical Center Quality Improvement Review Committee and was deemed exempt from approval by the Institutional Review Board.

Patients were included in the analysis if they were: 1) female, 2) newly diagnosed with non-metastatic, unilateral $\mathrm{BC}, 3) \leq 45$ years old, 4) had completed genetic counseling and GT, and 5) had completed surgical treatment of $\mathrm{BC}$ by the end of the study period. Patients were excluded if they had incomplete information regarding treatment, family history, or GT results.

The following data was collected via chart review: age; race and ethnicity; BC stage; type of breast surgery performed (lumpectomy, mastectomy, mastectomy with CRRM); and family history of BC and/or ovarian cancer (OC). Patients were considered to have a "significant" family history if they had at least one first- or second-degree 
TIMELY CANCER GENETIC COUNSELING AND TESTING YOUNG WOMEN WITH BREAST CANCER

relative with: 1) $\mathrm{OC}$ at any age, 2) $\mathrm{BC}$ diagnosed $\leq 30$ years old, 3) male $\mathrm{BC}$ at any age, or 4 ) $\geq 2$ relatives with $\mathrm{BC}$ at any age.

The specific GT performed was not standardized across the study cohort; rather, testing for BRCA1/2 or a multigene panel was tailored to each patient, based on their personal and family history of cancer, as well as patient preference. Results were: negative for a PV in a BC predisposition gene, positive for a PV in a BC predisposition gene, or uncertain (VUS). Given that the focus of this study was to evaluate the impact of GT results on BC risk management, PVs in genes that have not been shown to be associated with an increase in BC risks were considered to be negative. Of note, the NBN Slavic founder variant was included in analysis, as it was considered pathogenic during the study timeframe. Negative and VUS results were considered together as uninformative results.

Statistical analysis was performed using Chi-squared tests, Fisher's exact test, or t-tests, as appropriate, to examine the associations between surgical decisions (CRRM vs. no CRRM) and self-reported race/ethnicity, age, family history (significant vs. not significant), GT outcome (positive vs. uninformative), or BC stage ( $\leq$ II vs. III). Multivariate analysis was also performed to evaluate for any association between surgical decision and these factors. A $p$-value of $<0.05$ was considered to be statistically significant. Additionally, the association between age, family history, stage, and surgical decision was examined among patients with uninformative results. All analyses were performed using the SAS statistical software package (SAS/STAT software, Version 9.4 SAS Institute Inc., Cary, NC).

\section{RESULTS}

\section{Patient Population}

A total of 194 eligible patients were seen between November 2014 and June 2019 and included in this analysis. A total of 82 patients $(42.3 \%)$ had breast-conserving surgery (BCS), 45 (23.2\%) had unilateral mastectomy (UM), and 67 (34.5\%) had CRRM.

\section{Genetic Testing Results}

Regarding GT performed, 25 patients (12.9\%) had testing for $B R C A 1 / 2$, while $169(87.1 \%)$ had testing via a multigene panel. Pathogenic variants in $6 \mathrm{BC}$ genes were identified in $30(15.5 \%)$ patients: $B R C A 1(n=11$, $36.7 \%), \operatorname{BRCA2}(n=9,30 \%)$, CHEK2 $(n=6,20 \%$ overall; $n=1$ truncating PV, $n=5$ missense PV), NBN $(n=2$ Slavic founder heterozygotes, 6.7\%), $N F 1(n=1,3.3 \%)$, and $\operatorname{ATM}(n=1,3.3 \%$, Figure 1). Additionally, PVs in 
TIMELY CANCER GENETIC COUNSELING AND TESTING YOUNG WOMEN WITH BREAST CANCER

genes that have not been definitively associated with increased $\mathrm{BC}$ risks were identified in 2 patients and considered as a "negative" result for the purposes of this study (BRIP1, MUTYH heterozygote, $n=1$ each).

A total of $164(84.5 \%)$ women had uninformative GT results: $132(68 \%)$ negative, and $32(16.5 \%)$ with at least one VUS (Figure 1). Of the patients with a VUS, $18(56.3 \%)$ had a VUS in at least one BC gene $(A T M, n=6$; BRCA1, BRCA2, PALB2, PTEN, $n=2$ each; CHEK2, NF1, NBN, $n=1$ each; BARD1 and APC VUSs in same patient, $n=1)$. Additionally, $14(43.8 \%)$ had a VUS in a gene that has not been shown to be associated with BC predisposition (APC, POLD1, $n=3$ each; POLE MSH6, $n=2$ each; BRIP1, MLH1, RAD51C, and SDHB, $n=1$ each).

\section{Factors Associated with Surgical Decisions for the Whole Cohort}

The median age of the entire cohort was 39 (range 23-45, Table 1$)$. Most patients $(n=169,87.1 \%)$ were white; $19(9.8 \%)$ were Black, $5(2.6 \%)$ were Asian, and $1(0.5 \%)$ was of an unspecified race/ethnicity. Of the 67 patients who elected to have CRRM, $25(37.3 \%)$ had a PV in a moderate-risk BC predisposition gene $(C H E K 2, n=$ 4; $A T M, N B N$ heterozygote, $n=1$ each) or high-risk BC gene $(B R C A 1, n=11 ; B R C A 2, n=8$; Figure 2). Five $(16.7 \%)$ of the patients with a BC predisposition gene PV opted for a BCS/UM $(C H E K 2, n=2 ; B R C A 2, N B N$ heterozygote, $N F 1, n=1$ each) (Figure 2).

Compared with patients who chose a BCS/UM, patients who had a CRRM were younger (median age 37 vs. 39 years; $p=0.03)$, more likely to have a significant family history of BC/OC, $(35.8 \%$ vs. $15 \%$; $p<0.01)$, and similar stage distributions $(38.8 \%$ early stage vs. $31 \%$ late stage; $p=0.27)$. However, only a positive GT result was statistically significantly associated with CRRM in the multivariate analysis, with an OR of $11.4(95 \% \mathrm{CI}=3.95-$ 32.97, $p=<0.001)$ (Table 1).

\section{Factors Associated with Surgical Decisions in Patients with Uninformative Results}

Of the subset of 164 patients with uninformative results, $122(74.4 \%)$ opted for BCS/UM, and $42(25.6 \%)$ had a CRRM (Table 2; Figure 2). The median age at diagnosis for this group was 39 years (range 23-45 years). Most of the patients were white $(n=142,86.6 \%) ; 17(10.4 \%)$ were Black, $4(2.4 \%)$ were Asian, and $1(0.6 \%)$ was of an unspecified race/ethnicity. The genetic testing result (negative vs. VUS) was not observed to be associated with the decision to undergo CRRM. Of the 42 patients who elected to have CRRM, 12 had a VUS in any gene, while 20 of the 122 elected BCS/UM had a VUS in any gene $(28.6 \%$ vs. $16.4 \%, p=0.09)$. There was also 
no statistically significant difference in age at diagnosis (median age 38 vs. 39 years; $p=0.5$ ), significant family history $(26.2 \%$ vs. $14.7 \% ; p=0.09)$, or early disease stage $(45.2 \%$ vs. $30.6 \% ; p=0.08)$ between patients who opted for CRRM compared with those opted for BCS/UM among the subset of patients with an uninformative result (Table 2).

\section{DISCUSSION}

In this study, the identification of a $\mathrm{PV}$ in a $\mathrm{BC}$ predisposition gene was significantly associated with surgical decision for young women newly diagnosed with a BC. Patients with a PV in any BC predisposition gene were more likely to opt for CRRM at the time of surgery. Overall, $15.5 \%$ of patients were found to have a PV in a moderate- or high-risk $\mathrm{BC}$ predisposition gene, which is similar to the $12-18 \%$ rate previously reported in other premenopausal BC cohorts.[54-56]

Pathogenic variants in BRCA1/2 accounted for approximately two-thirds of PVs identified in this study, and most patients with a BRCA1/2 PV opted to have CRRM. Although there are limited data on long-term survival benefits, consideration of a CRRM is an established recommendation for women with a BRCA1/2 PV.[4, 23, 28, 29, 57] The rates of CRRM in our study was also similar to those reported previously for individuals with a BRCA1/2 PV who had been diagnosed with BC.[49, 51, 58]

In addition to $B R C A 1 / 2, \mathrm{PVs}$ in moderate-risk genes, such as $A T M$ and $C H E K 2$, may confer increased risks for a second primary BC. However, data on estimated risks are limited, and the long-term benefit of CRRM in this setting is uncertain. $[8,9,21-23,28,30,43,57]$ Despite this lack of data, more than half of the patients with a PV in a moderate-risk BC predisposition gene also opted to have CRRM, a rate similar to that previously reported among BC patients with a PV in a moderate-risk BC predisposition gene.[44-46] The number of patients identified to have a PV in a BC predisposition gene other than $B R C A 1 / 2$ in this study was small, which precluded our ability to adequately assess the association between the identification of a PV in a moderate-risk BC predisposition gene and the decision to pursue CRRM at the time of surgical BC treatment. However, patients with a $B R C A 1 / 2 \mathrm{PV}$ have been shown to be more likely than patients with PVs in other BC genes to choose CRRM.[44-46] Our study adds to the limited data available on surgical decisions on newly-diagnosed patients with a PV in a moderate-risk BC predisposition gene. 
TIMELY CANCER GENETIC COUNSELING AND TESTING YOUNG WOMEN WITH BREAST CANCER

Decisions regarding risk reducing surgical intervention are complex, and likely influenced by factors other than knowledge of a genetic predisposition.[59] The risks of developing another primary BC after the first diagnosis have been shown to be higher in patients with younger age at first diagnosis, or a family history of BC/OC.[14-18] Consequently, earlier age at diagnosis and family history of BC/OC have been shown to be positively correlated with the decision to have CRRM in women with BC, regardless of genetic testing outcome.[19, 37, 38, 46, 60-62] In this study, we did not observe any association between age at diagnosis and surgical decision when adjusted for GT result. However, all patients included in this study were 45 years old or younger, which hindered the ability to evaluate the impact of age at diagnosis on surgical decision making. Similarly, family history of BC and/or OC were not shown to be associated with a decision to have CRRM after adjustment for GT result in our study population.

Previous studies showing an association between family history of BC/OC and CRRM in women with BC either did not include information on GT, or only considered test results for BRCA1/2.[19, 36, 37, 60, 63-65] In our study, comprehensive GT could have identified patients with a BC predisposition who otherwise would have chosen CRRM based on family history. Furthermore, patients diagnosed with BC might choose to have CRRM based on their perception of a high risk for contralateral BC, especially when there is a significant family history.[66] Thus, the provision of pre-surgical genetic counseling and GT in this study may have helped to clarify estimated contralateral BC risks, and promoted risk-appropriate surgical decision-making among those without a genetic predisposition. Although higher CRRM rates with early-stage disease have previously been reported, disease stage was not shown to be associated with CRRM in our study.[19, 37, 61]

Although cancer GT can provide valuable information in risk assessment and promote risk-appropriate management, some test results have the potential to lead to unnecessary interventions. Variants of uncertain significance (VUSs) in cancer predisposition genes do not have any clinical implications, and should not be used in risk assessment or risk management considerations. With appropriate pre- and post-test counseling, patients with VUSs in BC predisposition genes did not elect to undergo CRRM more often than average-risk women.[45, 46, 50, 51, 67] However, interpretation of a VUS result can often be challenging, and high-risk management recommendations based on VUS findings, have been reported, including CRRM.[42, 49, 52, 53] As part of the multidisciplinary clinic, all patients in our study received pre- and post-test genetic counseling from a genetics specialist to discuss test implications and possible results. In our study, among those with an uninformative result, the CRRM rate was not significantly different between patients with a VUS in any gene, compared with patients 
with a negative result. Thus, incorporation of pre- and post-test genetic counseling as part of the multidisciplinary evaluation for young women newly diagnosed with BC could help ensure appropriate testing and accurate risk assessment.

Strengths of this study included systematic provision of genetic counseling to all patients. Additionally, all patients were seen by a genetic specialist. Limitations of this study included a small sample size of patients at one institution, which limits the ability to generalize the findings. While the type of GT offered was not standardized, the majority $(86.4 \%)$ were tested via a multigene panel. Finally, this exploratory study focused on the utility of genetic counseling and GT at the time of diagnosis for surgical decision-making among young patients with BC. Other factors that could potentially influence decision making regarding CRRM, such as socioeconomic status, reproductive planning, psychosocial factors, or concerns regarding potential cosmetic outcomes, were not explored.

\section{Conclusions}

For young women newly diagnosed with $\mathrm{BC}$ seen in a multidisciplinary clinic, the identification of a BC genetic predisposition was associated with the decision to pursue CRRM at the time of surgical BC treatment. Young patients with uninformative results had lower rates of CRRM, even among those with a significant family history. Thus, early incorporation of genetic services in the treatment planning process for young patients newly diagnosed with $\mathrm{BC}$ has the potential to lead to more appropriate risk assessment, which in turn could promote more risk-appropriate management. 


\section{REFERENCES}

1. Siegel RL, Miller KD, Jemal A (2020) Cancer statistics, 2020. CA: A Cancer Journal for Clinicians 70:730. https://doi.org/10.3322/caac. 21590

2. Beitsch PD, Whitworth PW, Hughes K, et al (2019) Underdiagnosis of hereditary breast cancer: Are genetic testing guidelines a tool or an obstacle? Journal of Clinical Oncology 37:453-460. https://doi.org/10.1200/JCO.18.01631

3. Tung N, Lin NU, Kidd J, et al (2016) Frequency of germline mutations in 25 cancer susceptibility genes in a sequential series of patients with breast cancer. Journal of Clinical Oncology 34:1460-1468. https://doi.org/10.1200/JCO.2015.65.0747

4. Kuchenbaecker KB, Hopper JL, Barnes DR, et al (2017) Risks of breast, ovarian, and contralateral breast cancer for BRCA1 and BRCA2 mutation carriers. JAMA - Journal of the American Medical Association 317:2402-2416. https://doi.org/10.1001/jama.2017.7112

5. Corso G, Montagna G, Figueiredo J, et al (2020) Hereditary Gastric and Breast Cancer Syndromes Related to CDH1 Germline Mutation: A Multidisciplinary Clinical Review. Cancers 12: https://doi.org/10.3390/cancers12061598

6. Ngeow J, Sesock K, Eng C (2017) Breast cancer risk and clinical implications for germline PTEN mutation carriers. Breast Cancer Research and Treatment 165:1-8

7. Hearle N, Schumacher V, Menko FH, et al (2006) Frequency and spectrum of cancers in the Peutz-Jeghers syndrome. Clinical Cancer Research 12:3209-3215. https://doi.org/10.1158/1078-0432.CCR-06-0083

8. Howell SJ, Hockenhull K, Salih Z, Evans DG (2017) Increased risk of breast cancer in neurofibromatosis type 1: Current insights. Breast Cancer: Targets and Therapy 9:531-536

9. Zhang G, Zeng Y, Liu Z, Wei W (2013) Significant association between Nijmegen breakage syndrome 1 657del5 polymorphism and breast cancer risk. Tumor Biology 34:2753-2757. https://doi.org/10.1007/s13277-013-0830-z

10. Yang X, Leslie G, Doroszuk A, et al (2020) Cancer Risks Associated With Germline PALB2 Pathogenic Variants: An International Study of 524 Families. Journal of clinical oncology : official journal of the American Society of Clinical Oncology 38:674-685. https://doi.org/10.1200/JCO.19.01907

11. Decker B, Allen J, Luccarini C, et al (2017) Rare, protein-truncating variants in ATM, CHEK2 and PALB2, but not XRCC2, are associated with increased breast cancer risks. Journal of medical genetics 54:732-741. https://doi.org/10.1136/JMEDGENET-2017-104588

12. Hall M, Bernhisel R, Hughes E, et al (2021) Germline Pathogenic Variants in the Ataxia Telangiectasia Mutated (ATM) Gene are Associated with High and Moderate Risks for Multiple Cancers. Cancer prevention research (Philadelphia, Pa) 14:433-440. https://doi.org/10.1158/1940-6207.CAPR-20-0448

13. Bougeard G, Renaux-Petel M, Flaman J-M, et al (2015) Revisiting Li-Fraumeni Syndrome From TP53 Mutation Carriers. Journal of clinical oncology : official journal of the American Society of Clinical Oncology 33:2345-52. https://doi.org/10.1200/JCO.2014.59.5728

14. Reiner AS, John EM, Brooks JD, et al (2013) Risk of asynchronous contralateral breast cancer in noncarriers of BRCA1 and BRCA2 mutations with a family history of breast cancer: A report from the women's environmental cancer and radiation epidemiology study. Journal of Clinical Oncology 31:433439. https://doi.org/10.1200/JCO.2012.43.2013

15. Lizarraga IM, Sugg SL, Weigel RJ, Scott-Conner CEH (2013) Review of risk factors for the development of contralateral breast cancer. American Journal of Surgery 206:704-708 
16. Akdeniz D, Schmidt MK, Seynaeve CM, et al (2019) Risk factors for metachronous contralateral breast cancer: A systematic review and meta-analysis. Breast 44:1-14

17. Giardiello D, Steyerberg EW, Hauptmann M, et al (2019) Prediction and clinical utility of a contralateral breast cancer risk model. Breast Cancer Research 21:. https://doi.org/10.1186/s13058-019-1221-1

18. Xiong Z, Yang L, Deng G, et al (2018) Patterns of Occurrence and Outcomes of Contralateral Breast Cancer: Analysis of SEER Data. Journal of Clinical Medicine 7:133. https://doi.org/10.3390/jcm7060133

19. Soran A, Kamali Polat A, Johnson R, McGuire KP (2014) Increasing trend of contralateral prophylactic mastectomy: What are the factors behind this phenomenon? Surgeon 12:316-322

20. Cybulski C, Kluźniak W, Huzarski T, et al (2015) Clinical outcomes in women with breast cancer and a PALB2 mutation: A prospective cohort analysis. The Lancet Oncology 16:638-644. https://doi.org/10.1016/S1470-2045(15)70142-7

21. Weischer M, Nordestgaard BG, Pharoah P, et al (2012) CHEK2*1100delC heterozygosity in women with breast cancer associated with early death, breast cancer-specific death, and increased risk of a second breast cancer. Journal of Clinical Oncology 30:4308-4316. https://doi.org/10.1200/JCO.2012.42.7336

22. Bernstein JL, Study W, Group C, Concannon P ATM, radiation, and the risk of second primary breast cancer. https://doi.org/10.1080/09553002.2017.1344363

23. Teoh V, Tasoulis MK, Gui G (2020) Contralateral prophylactic mastectomy in women with unilateral breast cancer who are genetic carriers, have a strong family history or are just young at presentation. Cancers 12

24. Plichta JK, Sebastian ML, Smith LA, et al (2019) Germline Genetic Testing: What the Breast Surgeon Needs to Know. Annals of Surgical Oncology 26:2184-2190. https://doi.org/10.1245/s10434-019-07341-8

25. Manahan ER, Kuerer HM, Sebastian M, et al (2019) Consensus Guidelines on Genetic Testing for Hereditary Breast Cancer from the American Society of Breast Surgeons. Annals of Surgical Oncology 26:3025-3031

26. Network NCC (2020) NCCN Clinical Practice Guidelines in Oncology: Breast Cancer Version 4.2020. https://www.nccn.org/professionals/physician_gls/pdf/breast.pdf. Accessed 12 Jun 2020

27. Network NCC (2020) NCCN Guidelines Version 1.2021: Genetic/Familial High-Risk Assessment : Breast, Ovarian, and Pancreatic. https://www.nccn.org/professionals/physician_gls/pdf/genetics_bop.pdf. Accessed 12 Sep 2020

28. Hunt KK, Euhus DM, Boughey JC, et al (2017) Society of Surgical Oncology Breast Disease Working Group Statement on Prophylactic (Risk-Reducing) Mastectomy. Annals of Surgical Oncology 24:375-397. https://doi.org/10.1245/s10434-016-5688-z

29. Hartmann LC, , T A Sellers, D J Schaid, T S Frank, C L Soderberg, D L Sitta, M H Frost, C S Grant, J H Donohue, J E Woods, S K McDonnell, C W Vockley, A Deffenbaugh, F J Couch RBJ (2001) Efficacy of Bilateral Prophylactic Mastectomy in BRCA1 and BRCA2 Gene Mutation Carriers. Journal of Clinical Oncology 93:1633-1637. https://doi.org/10.1093/jnci/93.21.1633

30. Boughey JC, Attai DJ, Chen SL, et al (2016) Contralateral Prophylactic Mastectomy (CPM) Consensus Statement from the American Society of Breast Surgeons: Data on CPM Outcomes and Risks. Annals of Surgical Oncology 23:3100-3105. https://doi.org/10.1245/s10434-016-5443-5

31. Boccardo C, Gentilini O (2016) Contralateral risk reducing mastectomy in patients with sporadic breast cancer. Benefits and hazards. European Journal of Surgical Oncology 42:913-918 
32. Carbine NE, Lostumbo L, Wallace J, Ko H (2018) Risk-reducing mastectomy for the prevention of primary breast cancer. Cochrane Database of Systematic Reviews 2018

33. Kenny R, Reed M, Subramanian A (2018) Mastectomy for risk reduction or symmetry in women without high risk gene mutation: A review. International Journal of Surgery 50:60-64

34. Tuttle TM, Habermann EB, Grund EH, et al (2007) Increasing use of contralateral prophylactic mastectomy for breast cancer patients: A trend toward more aggressive surgical treatment. Journal of Clinical Oncology 25:5203-5209. https://doi.org/10.1200/JCO.2007.12.3141

35. Tuttle TM, Jarosek S, Habermann EB, et al (2009) Increasing rates of contralateral prophylactic mastectomy among patients with ductal carcinoma in situ. Journal of Clinical Oncology 27:1362-1367. https://doi.org/10.1200/JCO.2008.20.1681

36. Nash R, Goodman M, Lin CC, et al (2017) State variation in the receipt of a contralateral prophylactic mastectomy amongwomen who received a diagnosis of invasive unilateral early-stage breast cancer in the United States, 2004-2012. JAMA Surgery 152:648-657. https://doi.org/10.1001/jamasurg.2017.0115

37. Jones NB, Wilson J, Kotur L, et al (2009) Contralateral prophylactic mastectomy for unilateral breast cancer: An increasing trend at a single institution. Annals of Surgical Oncology 16:2691-2696. https://doi.org/10.1245/s10434-009-0547-9

38. You Q, Chen K, Li Y, et al (2018) Factors associated with the increasing trend of contralateral prophylactic mastectomy among patients with ductal carcinoma in situ: Analysis of Surveillance, Epidemiology, and End Results data. Breast 40:147-155. https://doi.org/10.1016/j.breast.2018.05.001

39. Clerville JW (2018) Trends of Contralateral Prophylactic Mastectomy and Other Types of Breast Cancer Surgery. J Registry Manag 45:28-32

40. Couch FJ, Nathanson KL, Offit K (2014) Two Decades after BRCA: Setting Paradigms in Personalized Cancer Care and Prevention. Science 343:1466-70. https://doi.org/10.1126/science.1251827

41. Kurian AW, Hare EE, Mills MA, et al (2014) Clinical evaluation of a multiple-gene sequencing panel for hereditary cancer risk assessment. Journal of Clinical Oncology 32:2001-2009. https://doi.org/10.1200/JCO.2013.53.6607

42. Desmond A, Kurian AW, Gabree M, et al (2015) Clinical actionability of multigene panel testing for hereditary breast and ovarian cancer risk assessment. JAMA Oncology 1:943-951.

https://doi.org/10.1001/jamaoncol.2015.2690

43. Cragun D, Weidner A, Tezak A, et al (2020) Cancer risk management among female BRCA1/2, PALB2, CHEK2, and ATM carriers. Breast Cancer Research and Treatment 182:421-428. https://doi.org/10.1007/s10549-020-05699-y

44. Chang J, Seng S, Yoo J, et al (2019) Clinical Management of Patients at Risk for Hereditary Breast Cancer with Variants of Uncertain Significance in the Era of Multigene Panel Testing. Annals of Surgical Oncology 26:3389-3396. https://doi.org/10.1245/s10434-019-07595-2

45. Pederson HJ, Gopalakrishnan D, Noss R, et al (2018) Impact of Multigene Panel Testing on Surgical Decision Making in Breast Cancer Patients. Journal of the American College of Surgeons 226:560-565. https://doi.org/10.1016/j.jamcollsurg.2017.12.037

46. Elsayegh N, Webster RD, Gutierrez Barrera AM, et al (2018) Contralateral prophylactic mastectomy rate and predictive factors among patients with breast cancer who underwent multigene panel testing for hereditary cancer. Cancer Medicine 7:2718-2726. https://doi.org/10.1002/cam4.1519 
47. Richards S, Aziz N, Bale S, et al (2015) Standards and guidelines for the interpretation of sequence variants: a joint consensus recommendation of the American College of Medical Genetics and Genomics and the Association for Molecular Pathology. https://doi.org/10.1038/gim.2015.30

48. Mersch J, Brown N, Pirzadeh-Miller S, et al (2018) Prevalence of variant reclassification following hereditary cancer genetic testing. Journal of the American Medical Association 320:1266-1274. https://doi.org/10.1001/jama.2018.13152

49. Kurian AW, Li Y, Hamilton AS, et al (2017) Gaps in Incorporating Germline Genetic Testing Into Treatment Decision-Making for Early-Stage Breast Cancer. J Clin Oncol 35:2232-2239. https://doi.org/10.1200/JCO

50. Welsh JL, Hoskin TL, Day CN, et al (2017) Clinical Decision-Making in Patients with Variant of Uncertain Significance in BRCA1 or BRCA2 Genes. Annals of Surgical Oncology 24:3067-3072. https://doi.org/10.1245/s10434-017-5959-3

51. Morgan R, Brown A, Hamman K, Sampson J, Naik A MK (2018) Risk management decisions in women with BRCA1 and BRCA2 mutations. The American Journal of Surgery 15:899-903. https://doi.org/10.1016/j.amjsurg.2018.02.010

52. Macklin SK, Jackson JL, Atwal PS, Hines SL (2019) Physician interpretation of variants of uncertain significance. Familial Cancer 18:121-126. https://doi.org/10.1007/s10689-018-0086-2

53. Eccles BK, Copson E, Maishman T, et al (2015) Understanding of BRCA VUS genetic results by breast cancer specialists. BMC Cancer 15:936. https://doi.org/10.1186/s12885-015-1934-1

54. Buys SS, Sandbach JF, Gammon A, et al (2017) A study of over 35,000 women with breast cancer tested with a 25-gene panel of hereditary cancer genes. Cancer 123:1721-1730. https://doi.org/10.1002/cncr.30498

55. Tung N, Battelli C, Allen B, et al (2015) Frequency of mutations in individuals with breast cancer referred for BRCA 1 and BRCA 2 testing using next-generation sequencing with a 25-gene panel. Cancer 121:25-33. https://doi.org/10.1002/cncr.29010

56. Momozawa Y, Iwasaki Y, Parsons MT, et al (2018) Germline pathogenic variants of 11 breast cancer genes in 7,051 Japanese patients and 11,241 controls. Nature Communications 9:. https://doi.org/10.1038/s41467018-06581-8

57. Network NCC (2020) NCCN Guidelines Version 1.2020 Genetic/Familial High-Risk Assessment: Colorectal. https://www.nccn.org/professionals/physician_gls/pdf/genetics_colon.pdf. Accessed 19 Dec 2020

58. Flippo-Morton T, Walsh K, Chambers K, et al (2016) Surgical Decision Making in the BRCA-Positive Population: Institutional Experience and Comparison with Recent Literature. Breast Journal 22:35-44. https://doi.org/10.1111/tbj.12521

59. Sacks GD, Morrow M (2020) Addressing the Dilemma of Contralateral Prophylactic Mastectomy With Behavioral Science. Journal of Clinical Oncology 39:JCO.20.02239. https://doi.org/10.1200/jco.20.02239

60. Elsayegh N, Kuerer HM, Lin H, et al (2014) Predictors that influence contralateral prophylactic mastectomy election among women with ductal carcinoma in situ who were evaluated for BRCA genetic testing. Annals of Surgical Oncology 21:3466-3472. https://doi.org/10.1245/s10434-014-3747-x

61. Metcalfe KA, Retrouvey H, Kerrebijn I, et al (2019) Predictors of uptake of contralateral prophylactic mastectomy in women with nonhereditary breast cancer. Cancer cncr.32405.

https://doi.org/10.1002/cncr.32405 
62. Nash R, Goodman M, Lin CC, et al (2017) State variation in the receipt of a contralateral prophylactic mastectomy among women who received a diagnosis of invasive unilateral early-stage breast cancer in the United States, 2004-2012. JAMA Surgery 152:648-657. https://doi.org/10.1001/jamasurg.2017.0115

63. Metcalfe KA, Retrouvey H, Kerrebijn I, et al (2019) Predictors of uptake of contralateral prophylactic mastectomy in women with nonhereditary breast cancer. Cancer. https://doi.org/10.1002/cncr.32405

64. You Q, Chen K, Li Y, et al (2018) Factors associated with the increasing trend of contralateral prophylactic mastectomy among patients with ductal carcinoma in situ: Analysis of Surveillance, Epidemiology, and End Results data. Breast 40:147-155. https://doi.org/10.1016/j.breast.2018.05.001

65. Yi M, Hunt KK, Arun BK, et al (2010) Factors affecting the decision of breast cancer patients to undergo contralateral prophylactic mastectomy. Cancer Prevention Research 3:1026-1034. https://doi.org/10.1158/1940-6207.CAPR-09-0130

66. Abbott A, Rueth N, Pappas-Varco S, et al (2011) Perceptions of contralateral breast cancer: An overestimation of risk. In: Annals of Surgical Oncology. Ann Surg Oncol, pp 3129-3136

67. Culver J, Brinkerhoff C, Clague J, et al (2013) Variants of uncertain significance in BRCA testing: Evaluation of surgical decisions, risk perception, and cancer distress. Clinical Genetics 84:464-472. https://doi.org/10.1111/cge.12097 
TIMELY CANCER GENETIC COUNSELING AND TESTING YOUNG WOMEN WITH BREAST CANCER

\section{STATEMENTS AND DECLARATIONS}

Funding: This research project was supported in part by funding from the Hackers for Hope Pittsburgh, a 501c(3) charity.

Competing interests: The authors have no relevant financial or non-financial interests to disclose.

Author contributions: SAD collected, analyzed, and interpreted the data, and prepared the manuscript; DLT interpreted the data and participated in manuscript preparation; PFM, JGS, and RRJ contributed to clinical data collection, and reviewed the manuscript; EJD conceived and designed the study, provided clinical data, critically reviewed the data, and reviewed the manuscript, PLM conceived and designed the study, analyzed and interpreted the data, and prepared the manuscript. All authors approved the final manuscript.

Data availability: The datasets generated during and/or analyzed during the current study are not publicly available in order to protect patient privacy; however, a de-identified dataset is available from author PLM upon reasonable request.

Ethics approval: This study was performed in line with the principles of the Declaration of Helsinki. The project was reviewed and approved by the University of Pittsburgh Medical Center Quality Improvement Review Committee and was deemed exempt from approval by the Institutional Review Board.

Consent to participate: This was a quality control study. The University of Pittsburgh Medical Center Quality Improvement Review Committee and Institutional Review Board has confirmed that informed consent was not required.

Consent to publish: Not applicable; no identifying information is included in this report or associated data, figures, or tables. 


\section{FIGURE LEGENDS}

Fig 2

\section{Breast cancer (BC) gene VUS:}

CRRM: $n=1$ each ATM, BRCA2, CHEK2, NF1, PTEN

BCS/UM: ATM, $n=5 ; n=2$ each, BRCA1, PALB2; $n=1$ each, APC/BARD1, BRCA2, NBN, PTEN

\section{Other gene VUS:}

CRRM: POLE, $n=2 ; n=1$ each APC, BRIPI, MLH1, RAD51C, POLD1

BCS/UM: $n=2$ each APC, MSH6, POLD1; SDHB, $n=1$

\section{FIGURE DESCRIPTIVE CAPTIONS}

\section{Fig 1}

A pie chart indicating that $123(68 \%)$ of patients had a negative genetic testing result, $32(16.5 \%)$ had a variant of uncertain significance, and $30(15.5 \%)$ had a positive result. A second pie chart indicates that of the 30 patients with a positive result, $11(36.7 \%)$ had a $B R C A 1 \mathrm{PV} ; 9(30 \%)$ had a BRCA2 PV; $6(20 \%)$ had a CHEK2 PV; $2(6.7 \%)$ had an $N B N$ variant; 1 (3.2\%) had an ATM PV; and 1 (3.3\%) had an NFI PV.

\section{Fig 2}

A bar graph indicating that of the 30 patients with a PV, 83.3\% had a CRRM. A pie chart leading from this part of the bar graph indicates that of patients with a PV who had a CRRM, 11 (44\%) had a BRCAl PV; 8 (32\%) had a BRCA2 PV; 4 (16\%) had a CHEK2 PV; 1 (4\%) had an ATM PV; and 1 (45) had an NBN variant.

The remaining $16.7 \%$ of patients with a PV had a BCS or UM. A pie chart leading from this part of the bar graph indicates that $2(40 \%)$ had a CHEK2 PV; $1(20 \%)$ had a BRCA2 PV; $1(20 \%)$ had an NF1 PV; and $1(20 \%)$ had an $N B N$ variant.

The remainder of the bar graph indicates that of patients with a VUS in a BC gene, 27.8\% had a CRRM, while 76.5\% had a BCS/UM. Of patients with a VUS in any other gene, 50\% had a CRRM, and 50\% had a BCS/UM. Finally, of patients with negative test results, $23.1 \%$ had a CRRM, and $77.3 \%$ had a BCS/UM. 


\section{Figures}

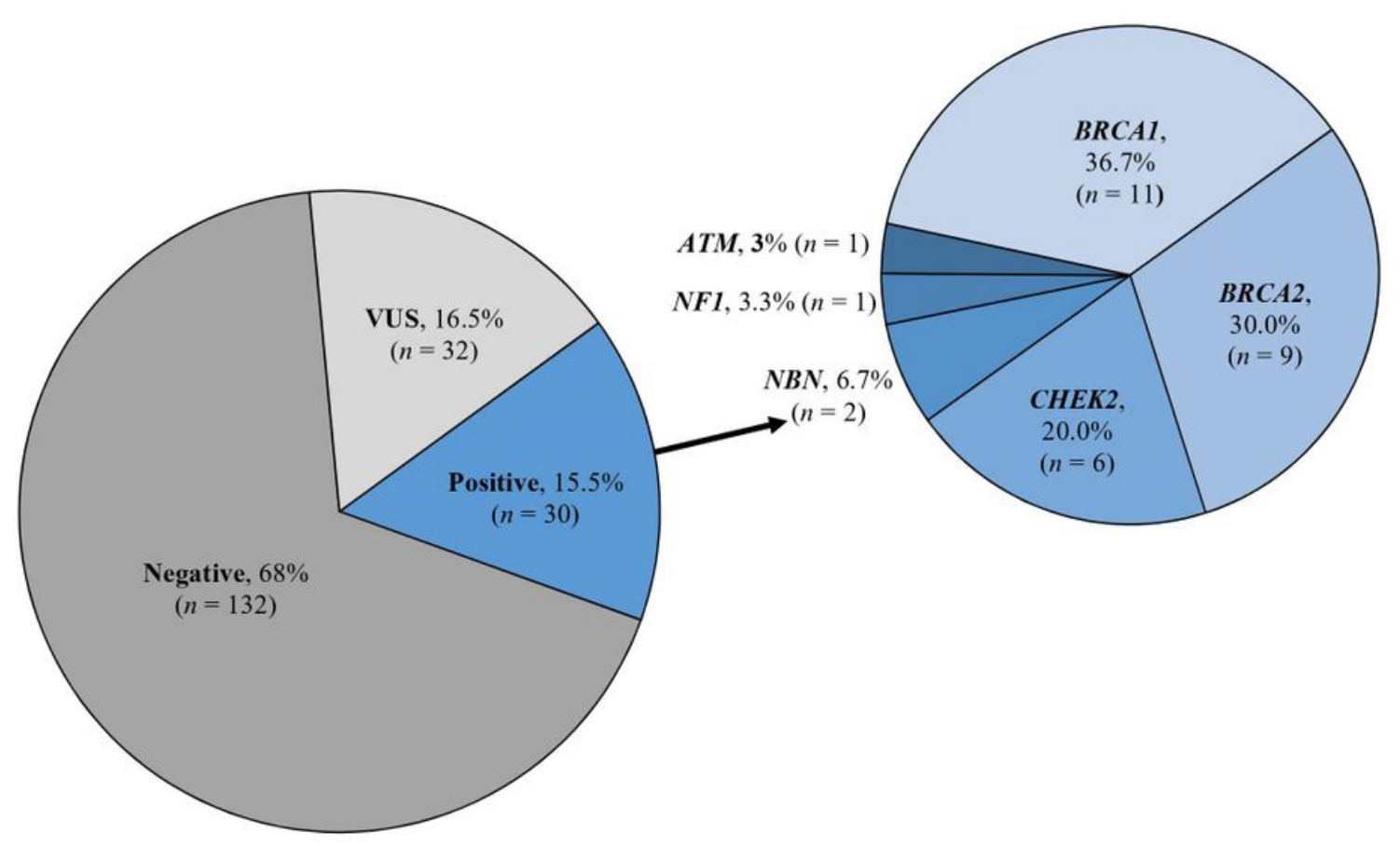

\section{Figure 1}

Genetic Testing Outcomes in All Patients $(n=194)$

A pie chart indicating that $123(68 \%)$ of patients had a negative genetic testing result, $32(16.5 \%)$ had a variant of uncertain significance, and $30(15.5 \%)$ had a positive result. A second pie chart indicates that of the 30 patients with a positive result, $11(36.7 \%)$ had a BRCA1 PV; $9(30 \%)$ had a BRCA2 PV; $6(20 \%)$ had a CHEK2 PV; 2 (6.7\%) had an NBN variant; 1 (3.2\%) had an ATM PV; and 1 (3.3\%) had an NF1 PV. 


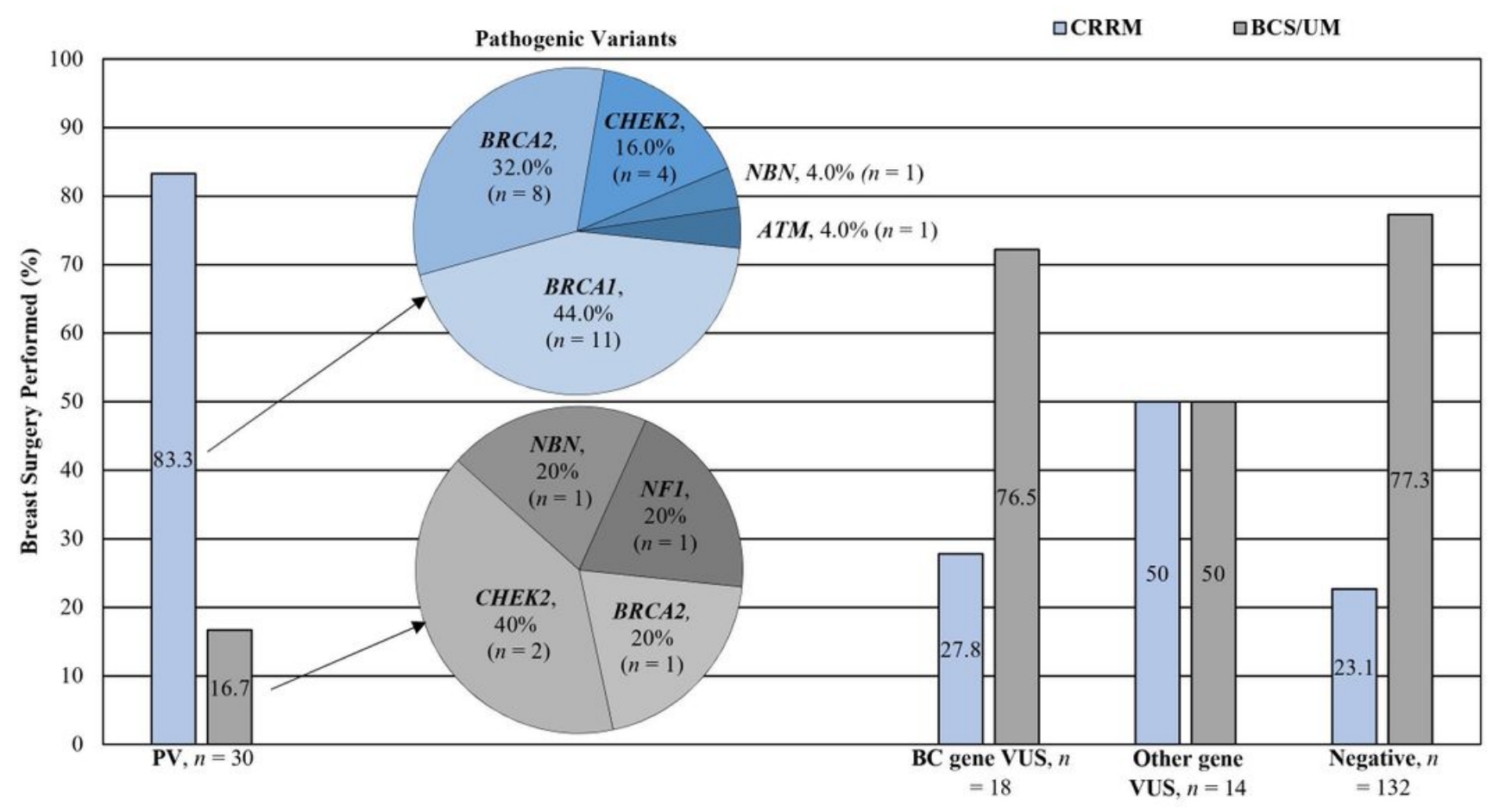

Figure 2

Overall Genetic Testing Results and Surgical Decision-Making Outcomes ( $\mathrm{n}=194)$

Breast cancer (BC) gene VUS: CRRM: $n=1$ each ATM, BRCA2, CHEK2, NF1, PTEN BCS/UM: ATM, $n=5 ; n$ $=2$ each, BRCA1, PALB2; $n=1$ each, APC/BARD1, BRCA2, NBN, PTEN

Other gene VUS: CRRM: POLE, $n=2 ; n=1$ each APC, BRIP1, MLH1, RAD51C, POLD1 BCS/UM: $n=2$ each APC, MSH6, POLD1; SDHB, $\mathrm{n}=1$

A bar graph indicating that of the 30 patients with a PV, 83.3\% had a CRRM. A pie chart leading from this part of the bar graph indicates that of patients with a PV who had a CRRM, 11 (44\%) had a BRCA1 PV; 8 (32\%) had a BRCA2 PV; 4 (16\%) had a CHEK2 PV; 1 (4\%) had an ATM PV; and 1 (45) had an NBN variant.

The remaining $16.7 \%$ of patients with a PV had a BCS or UM. A pie chart leading from this part of the bar graph indicates that $2(40 \%)$ had a CHEK2 PV; 1 (20\%) had a BRCA2 PV; $1(20 \%)$ had an NF1 PV; and 1 $(20 \%)$ had an NBN variant.

The remainder of the bar graph indicates that of patients with a VUS in a BC gene, $27.8 \%$ had a CRRM, while $76.5 \%$ had a BCS/UM. Of patients with a VUS in any other gene, 50\% had a CRRM, and $50 \%$ had a BCS/UM. Finally, of patients with negative test results, $23.1 \%$ had a CRRM, and $77.3 \%$ had a BCS/UM. 


\section{Supplementary Files}

This is a list of supplementary files associated with this preprint. Click to download.

- BCRTTable1.pdf

- BCRTTable2.pdf 